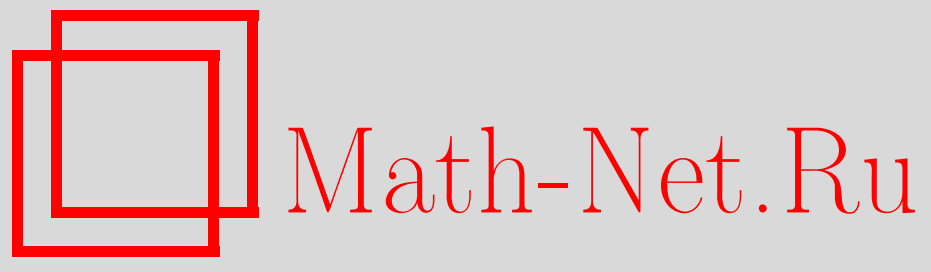

Д. Д. Киселев, Поля разложения конечных групп, Изв. РАН. Сер. матем., 2012, том 76, выпуск 6, 95-106

DOI: https://doi.org/10.4213/im7331

Использование Общероссийского математического портала Math-Net.Ru подразумевает, что вы прочитали и согласны с пользовательским соглашением http: //www . mathnet.ru/rus/agreement

Параметры загрузки:

IP: 52.90 .164 .192

26 апреля 2023 г., 08:37:40

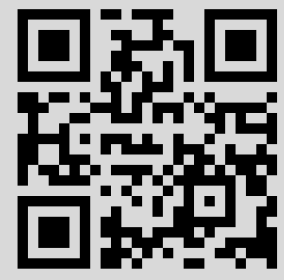




\title{
Д. Д. Киселев
}

\section{Поля разложения конечных групп}

\begin{abstract}
Дано более простое доказательство теоремы Голдшмидта-Айзекса для случая $p>2$. Приведены новые достаточные условия применимости теоремы Голдшмидта-Айзекса для случая $p=2$. Как следствие, доказана теорема об оценке индекса Шура произвольного неприводимого комплексного представления конечной группы над полем рациональных чисел. Из доказательства указанной теоремы об оценке выведен факт о том, что в практических приложениях нет необходимости проверять достаточные условия применимости теоремы Голдшмидта-Айзекса для случая $p=2$, их заведомо можно считать выполненными. Также доказана теорема о связи реализуемости любого комплексного представления конечной группы нечетного порядка специального типа над полем рациональных чисел и возможности построения правильных многоугольников циркулем и линейкой.
\end{abstract}

Библиография: 6 наименований.

Ключевые слова: конечная группа, представление конечной группы, индекс Шура.

\section{§ 1. Введение}

1.1. История вопроса. Теория полей разложения конечных групп имеет свое начало, пожалуй, от фундаментальной теоремы Брауэра о реализуемости любого комплексного представления произвольной конечной группы экспоненты $n$ в поле $\mathbb{Q}(\sqrt[n]{1})$. Данный результат послужил толчком для дальнейших исследований. А именно, было доказано, что любое абсолютно неприводимое представление произвольной конечной группы $G$ над алгебраически замкнутым полем ненулевой характеристики $p$ реализуется в поле $\mathbb{F}_{p}(\chi)$, где $\chi$ - характер представления. Этот факт оказался тесно связан с теоремой Веддербарна о том, что всякое конечное тело является полем.

Поэтому возникла задача описания минимальных полей разложения произвольной конечной группы $G$ в нулевой характеристике. Было введено фундаментальное понятие индекса Шура как некой числовой характеристики "расстояния" поля разложения абсолютно неприводимого характера $\chi$ относительно фиксированного поля, содержащего все значения характера $\chi$. Были доказаны теоремы о свойствах индекса Шура над произвольным полем и полными полями, установлена связь индекса Шура над глобальным полем с индексами Шура над пополнениями этого поля.

Следующая теорема Шиллинга-Витта-Рокетта (см. [1, теорема 41.9]) практически полностью решает проблему индекса Шура для конечных $p$-групп. 
Теорема 1.1 (О. Шиллинг, И. Витт, П. Рокетт). Пусть G - конечная р-группа, $K$ - поле нулевой характеристики. Тогда для любого характера $\chi \in \operatorname{Irr} G$ выполнено $m_{K}(\chi)=1$, если $p>2$. Если же $p=2$, то $m_{K}(\chi) \leqslant 2$.

Некоторым обобщением теоремы 1.1 является теорема Фонга. Ее доказательство изложено, например, в [2, теорема 4.2.5].

Теорема 1.2 (П. Фонг). Пусть $G$ - конечная группа порядка $n=p^{s} m$, где $p$ - нечетное простое число, причем $(m, p)=1$. Тогда для любого характера $\chi \in \operatorname{Irr} G$ выполнено $m_{K(\sqrt[m]{1})}(\chi)=1$, где $K$ - поле нулевой характеристики.

Легко видеть, что теоремы Фонга и Шиллинга-Витта-Рокетта связаны тем, что группа Галуа $\operatorname{Gal}(K(\sqrt[|G|]{1}) / K)$ в них оказывается циклической. В обобщение этой идеи была доказана следующая (см. [3, теорема 1])

Теорема 1.3 (Д. М. Голдшмидт, И. М. Айзекс). Пусть $G$ - конечная груnпа экспоненты $n, K$ - любое поле нулевой характеристики. Пусть $\operatorname{Gal}(K(\sqrt[n]{1}) / K)$ - ииклическая р-группа. Если $p=2$, дополнительно предположим, что $\sqrt{-1} \in K$. Тогда для любого характера $\chi \in \operatorname{Irr} G$ выполнено $m_{K}(\chi)=1$.

1.2. Результаты работы. В настоящей работе дается более простое доказательство теоремы Голдшмидта-Айзекса для случая $p>2$ с применением теоремы Фонга, доказательство которой изложено независимо от теоремы Голдшмидта-Айзекса в [2, теорема 4.2.5]. Заметим, что оригинальное доказательство теоремы Голдшмидта-Айзекса, полученное в [3], не опирается на теорему Фонга. Вместо этого теорема Фонга получается как простое следствие.

В работе получены новые достаточные условия применимости теоремы Голдшмидта-Айзекса в случае $p=2$. А именно, условие принадлежности элемента $\sqrt{-1}$ основному полю несколько ослаблено.

ТЕОРемА 1.4 (алгоритмический признак). Пусть $G$ - конечная группа экспоненты $n, K$ - произвольное поле нулевой характеристики. Обозначим через $F$ поле в башне $\mathbb{Q} \subset F \subset \mathbb{Q}(\sqrt[n]{1})$ с условием $\operatorname{Gal}(K(\sqrt[n]{1}) / K) \cong \operatorname{Gal}(\mathbb{Q}(\sqrt[n]{1}) / F)$. Предположим, что при каноническом вложении группы $\operatorname{Gal}(K(\sqrt[n]{1}) / K)$ в группу $\mathbb{Z}_{n}^{*}$ выполняется условие $\operatorname{Gal}(K(\sqrt[n]{1}) / K) \nsubseteq A_{2}$, где $A_{2}-$ подгруппа в $\mathbb{Z}_{n}^{*}$, изоморфная $\mathbb{Z}_{2^{\nu_{2}(n)}}^{*}$. Если $\operatorname{Gal}(K(\sqrt[n]{1}) / K)$ - ииклическая группа, причем $F \nsubseteq \mathbb{R}$, то для любого характера $\chi \in \operatorname{Irr} G$ выполнено $m_{K}(\chi)=1$.

Теорема 1.5. Пусть $G$ - конечная группа экспоненты $n, K$ - произвольное поле нулевой характеристики. Построим поле $F$ по полю $K$ согласно формулировке алгоритмического признака. Пусть $\operatorname{Gal}(K(\sqrt[n]{1}) / K)$ - ииклическая гpynna, $F \nsubseteq \mathbb{R} u$

$$
\nu_{2}\left(\min \left\{t \in \mathbb{N} \mid \frac{2^{t}-1}{n_{2^{\prime}}} \in \mathbb{N}\right\}\right)=\nu_{2}\left(\varphi\left(n_{2^{\prime}}\right)\right) .
$$

Тогда для любого характера $\chi \in \operatorname{Irr} G$ выполнено $m_{K}(\chi)=1$.

Заметим, что в формулировках теорем $1.4,1.5$ поле $F$ можно заменить на поле $F_{1}$, отвечающее силовской 2-подгруппе в $\operatorname{Gal}(\mathbb{Q}(\sqrt[n]{1}) / F)$. 
Следующая теорема дает оценку индекса Шура неприводимого комплексного характера $\chi$ конечной группы $G$ над полем рациональных чисел $\mathbb{Q}$. Указанная оценка "равномерна" по всем конечным группам экспоненты $n$ или по всем конечным группам порядка $n$.

Теорема 1.6 (об оценке). Пусть $G$ - конечная группа порядка (или экспоненть) $n$. Пусть $m$ - порядок какой-нибудь максимальной ииклической подгруппь в группе $\mathbb{Z}_{n}^{*}$. Если $n \neq 4$, mо $m_{\mathbb{Q}}(\chi) \mid \frac{\varphi(n)}{m}$ для любого $\chi \in \operatorname{Irr} G$.

Также устанавливается следующий результат.

Теорема 1.7. Пусть $G$ - конечная группа экспоненты $n$. Пусть $n=$ $p_{1}^{k_{1}} \cdots p_{s}^{k_{s}}$ - каноническое разложение числа $n$ на простые множители. Положим $m=p_{1} \cdots p_{s}$. Если $2 \nmid m$ и правильный $m$-угольник допускает построение ииркулем и линейкой, то для любого характера $\chi \in \operatorname{Irr} G$ выполнено $m_{\mathbb{Q}}(\chi)=1$.

1.3. Обозначения. Обозначения во многом стандартны и поясняются по ходу работы. Тем не менее, во избежание недопонимания, приведем некоторые из них: $m_{K}(\chi)$ - индекс Шура неприводимого комплексного характера $\chi$ конечной группы $G$ относительно поля $K$ (при этом всегда будет предполагаться, что характеристика поля $K$ нулевая); $\varepsilon_{n}-$ примитивный корень степени $n$ из единицы в алгебраическом замыкании поля рациональных чисел $\mathbb{Q} ; \bar{K}$ - алгебраическое замыкание поля $K$ или же переход к полю вычетов в зависимости от контекста; $K \cdot L$ - композит полей алгебраических чисел $K$ и $L$ в поле $\overline{\mathbb{Q}}$; $(A: B)$ - индекс алгебраической подструктуры $B$ в алгебраической структуpe $A$ (например, запись $\left(\mathbb{Q}\left(\varepsilon_{n}\right): \mathbb{Q}\right)$ обозначает степень поля $\mathbb{Q}\left(\varepsilon_{n}\right)$ над $\mathbb{Q}$ ); $\mathbb{Z}_{n}$ - кольцо классов вычетов по модулю $n$ или произвольный представитель класса циклических групп порядка $n ; \mathbb{Z}_{n}^{*}-$ группа обратимых по умножению элементов кольца вычетов $\mathbb{Z}_{n} ; R^{*}$ - мультипликативная группа ассоциативного кольца $R$ с единицей; $R_{K}$ - кольцо целых алгебраических чисел поля алгебраических чисел $K ; K_{P}-P$-адическое пополнение поля алгебраических чисел $K$, где $P$ - либо конечный, либо бесконечный простой идеал кольца $R_{K}$; $B(K)$ - группа Брауэра поля $K ; B(L / K)$ - подгруппа группы $B(K)$, состоящая из расщепляемых в расширении $L / K$ классов центрально-простых $K$-алгебр; $\operatorname{inv}_{K_{P}}[A]$ - инвариант Хассе класса $[A] \in B\left(K_{P}\right)$; $\operatorname{index}_{K}[A]$ - индекс класса алгебр $[A] \in B(K)$ (поле $K$ в обозначении индекса может не указываться); $f(A / B)$ - степень инерции локального поля $A$ над своим локальным подполем $B ; e(A / B)$ - индекс ветвления локального поля $A$ над своим локальным подполем $B ;\langle X\rangle$ - подгруппа, порожденная множеством $X ; N_{L / K}(L)$ - множество элементов поля $K$, представимых в виде нормы элементов из поля $L$ (предполагается, что расширение $L / K$ является конечным нормальным и сепарабельным расширением); $\nu_{p}(n)$ - кратность простого числа $p$ в каноническом разложении натурального числа $n$ на простые множители; $n_{p}-p$-часть натурального числа $n$, где $p$ - простое число; $n_{p^{\prime}}-p^{\prime}$-часть натурального числа $n$, где $p$ - простое число.

Запись $i \in \overline{m, n}$ означает, что натуральный индекс $i$ пробегает в порядке возрастания множество натуральных чисел от $m$ до $n$ включительно. Предполагается, конечно, что $m \leqslant n$. 


\section{§ 2. Доказательство теорем}

2.1. Доказательство теоремы Голдшмидта-Айзекса. Приведем более простое доказательство теоремы 1.3 для случая $p>2$.

Фиксируем произвольную конечную группу $G$ порядка $n=p_{1}^{k_{1}} \cdots p_{s}^{k_{s}}$. Также фиксируем $\chi \in \operatorname{Irr} G$. Докажем, что $m_{K}(\chi)=1$. Обозначим, далее, через $A(K, \chi)$ простую компоненту групповой алгебры $K G$, соответствующую характеру $\chi$. Сначала заметим, что поле нулевой характеристики $K$, фигурирующее в теореме 1.3 , можно считать вложенным в $\mathbb{Q}\left(\varepsilon_{n}\right)$. Можно также считать, что $\varepsilon_{n}=e^{2 \pi i / n}$. Действительно, пусть $K$ - произвольное поле нулевой характеристики; тогда $\operatorname{Gal}\left(K\left(\varepsilon_{n}\right) / K\right)$ является с точностью до изоморфизма подгруппой в $\operatorname{Gal}\left(\mathbb{Q}\left(\varepsilon_{n}\right) / \mathbb{Q}\right) \cong \mathbb{Z}_{n}^{*}$. В силу фундаментального соответствия Галуа найдется единственное (с точностью до изоморфизма) поле $F$ такое, что $\mathbb{Q} \subset F \subset \mathbb{Q}\left(\varepsilon_{n}\right)$, причем $\operatorname{Gal}\left(K\left(\varepsilon_{n}\right) / K\right) \cong \operatorname{Gal}\left(\mathbb{Q}\left(\varepsilon_{n}\right) / F\right)$. Более того, $F=\mathbb{Q}(\theta)$ в силу теоремы о примитивном элементе. В частности, найдется многочлен $f(x) \in \mathbb{Q}[x]$ такой, что $\theta=f\left(\varepsilon_{n}\right)$. Поскольку $\theta^{\sigma}=\theta$ для любого $\sigma \in \operatorname{Gal}\left(\mathbb{Q}\left(\varepsilon_{n}\right) / F\right)$, то в силу того, что $\sigma$ действует на $\mathbb{Q}$ тождественно, а на $\varepsilon_{n}-$ как возведение в некоторую степень, следует $\theta^{\sigma}=\theta$ для любого $\sigma \in \operatorname{Gal}\left(K\left(\varepsilon_{n}\right) / K\right)$, так как можно считать $\mathbb{Q} \subset K$. Итак, $F \subset K$.

Если теперь теорема 1.3 доказана для поля $F$, то, очевидно, она доказана и для $K$. Поэтому далее до конца доказательства будем считать, что $K$ - поле алгебраических чисел, лежащее в $\mathbb{Q}\left(\varepsilon_{n}\right)$.

Напомним, что по условию $\operatorname{Gal}\left(K\left(\varepsilon_{n}\right) / K\right)$ является циклической $p$-группой при $p>2$. Поскольку решетка подгрупп конечной циклической $p$-группы является линейно упорядоченным по включению множеством, из фундаментального соответствия Галуа следует, что все поля в цепочке $K \subset K\left(\varepsilon_{n}\right)$ также представляют линейно упорядоченное множество по включению. Можно поэтому считать, что

$$
K \subseteq K\left(\varepsilon_{p_{1}^{k_{1}}}\right) \subseteq K\left(\varepsilon_{p_{2}^{k_{2}}}\right) \subseteq \cdots \subseteq K\left(\varepsilon_{p_{1}^{k_{1}}}\right) \subseteq K\left(\varepsilon_{n}\right)=\mathbb{Q}\left(\varepsilon_{n}\right) .
$$

Докажем, что на самом деле $K\left(\varepsilon_{p_{1}^{k_{1}}}\right)=K\left(\varepsilon_{n}\right)$. Рассмотрим произведение $\varepsilon_{p_{1}^{k_{1}}} \cdots \varepsilon_{p_{s}^{k_{s}}}$ и заметим, что

$$
\varepsilon_{p_{j}^{k_{j}}}=\varepsilon_{n}^{n_{p_{j}^{\prime}}}=\exp \left\{\frac{2 \pi i}{p_{j}^{k_{j}}}\right\}
$$

поэтому

$$
\varepsilon_{p_{1}^{k_{1}}} \cdots \varepsilon_{p_{s}^{k_{s}}}=\exp \left\{2 \pi i \frac{\sum_{j=1}^{s} p_{1}^{k_{1}} \cdots p_{j-1}^{k_{j-1}} p_{j+1}^{k_{j+1}} \cdots p_{s}^{k_{s}}}{n}\right\} .
$$

Однако число $\sum_{j=1}^{s} p_{1}^{k_{1}} \cdots p_{j-1}^{k_{j-1}} p_{j+1}^{k_{j+1}} \cdots p_{s}^{k_{s}}$ взаимно просто с $n$. Это означает, что поле $K\left(\varepsilon_{p_{1}}^{k_{1}}\right)$ содержит некоторый примитивный корень степени $n$ из единицы. В силу нормальности расширения $K\left(\varepsilon_{p_{1}}^{k_{1}}\right) / K$ заключаем, что $K\left(\varepsilon_{p_{1}^{k_{1}}}\right)=K\left(\varepsilon_{n}\right)=\mathbb{Q}\left(\varepsilon_{n}\right)$.

Теперь в силу теоремы Хассе-Брауэра-Нётер-Альберта [1, теорема 32.11] достаточно доказать, что

$$
\operatorname{index}_{K_{P}}\left[K_{P} \otimes_{K} A(K, \chi)\right]=1
$$


для любого простого идеала $P$ кольца $R_{K}$ целых алгебраических чисел поля $K$, включая бесконечные (так как $m_{K}(\chi)=m_{K(\chi)}(\chi)$, без ограничения общности можно считать, что $K=K(\chi))$. Если $P$ - бесконечный комплексный идеал, то index $_{K_{P}}\left[K_{P} \otimes_{K} A(K, \chi)\right]=1$. Если $P$ - вещественный идеал, то

$$
\operatorname{index}_{K_{P}}\left[K_{P} \otimes_{K} A(K, \chi)\right] \mid 2 .
$$

Однако легко видеть, что $\operatorname{Gal}\left(K_{P}\left(\varepsilon_{n}\right) / K_{P}\right)$ является $p$-группой, но $p>2$, a

$$
\operatorname{index}_{K_{P}\left(\varepsilon_{n}\right)}\left[K_{P}\left(\varepsilon_{n}\right) \otimes_{K_{P}}\left(K_{P} \otimes_{K} A(K, \chi)\right)\right]=1 .
$$

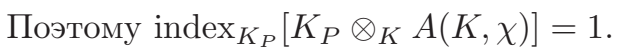

Значит, достаточно ограничиться рассмотрением лишь неархимедовых пополнений поля $K$. Возьмем какое-нибудь простое число $q \in\left\{p_{i} \mid i=\overline{1, s-1}\right\}$ и выберем любой простой идеал $\mathfrak{p} \triangleleft R_{K}$, содержащий $q \mathbb{Z}$.

Докажем, что расширение $K\left(\varepsilon_{p_{1}^{k_{1}}}\right) / K$ неразветвлено по p. Действительно, как мы знаем, в расширении $K_{\mathfrak{p}}\left(\varepsilon_{p_{1}^{k_{1}}}\right) / K_{\mathfrak{p}}$ существует единственное поле инерции $W$ :

$$
K_{\mathfrak{p}} \subseteq W \subseteq K_{\mathfrak{p}}\left(\varepsilon_{p_{1}^{k_{1}}}\right) .
$$

Поскольку $f\left(K_{\mathfrak{p}}\left(\varepsilon_{p_{1}^{k_{1}}}\right) / W\right)=1$, в силу сепарабельности многочлена $x^{p_{s}^{k_{s}}}-1 \in$ $\mathbb{F}_{q}[x]$ и леммы Гензеля получаем, что $W=K_{\mathfrak{p}}\left(\varepsilon_{p_{1}}\right)$. Это и означает неразветвленность расширения $K_{\mathfrak{p}}\left(\varepsilon_{p_{1}^{k_{1}}}\right) / K_{\mathfrak{p}}$.

Известно, что $A(K, \chi)$ - центрально-простая компонента групповой алгебры $K G$. Более того,

$$
[A(K, \chi)]=\left[\left(K\left(\varepsilon_{p_{1}^{k_{1}}}\right) / K, f\right)\right]
$$

в группе $B\left(K\left(\varepsilon_{p_{1}^{k_{1}}}\right) / K\right)$, где

$$
f: \operatorname{Gal}\left(K\left(\varepsilon_{p_{1}^{k_{1}}}\right) / K\right) \times \operatorname{Gal}\left(K\left(\varepsilon_{p_{1}^{k_{1}}}\right) / K\right) \rightarrow K\left(\varepsilon_{p_{1}^{k_{1}}}\right)^{*} .
$$

В силу доказательства теоремы Бенарда-Шахера (см. [4, теорема 74.20]) можно считать, что $\operatorname{Im} f \subseteq\left\langle\varepsilon_{n}\right\rangle$. Однако любая скрещенная алгебра $(L / K, f)$ в случае циклического расширения Галуа $L / K$ является циклической. В рассматриваемом случае это означает, что

$$
[A(K, \chi)]=\left[\left(K\left(\varepsilon_{p_{1}^{k_{1}}}\right) / K, f\right)\right]=\left[\left(K\left(\varepsilon_{p_{1}^{k_{1}}}\right) / K, \sigma, a\right)\right],
$$

где $\sigma$ порождает группу $\operatorname{Gal}\left(K\left(\varepsilon_{p_{1}^{k_{1}}}\right) / K\right)$, а элемент $a=\prod_{j=0}^{p^{r}-1} f_{\sigma^{j}, \sigma}$ принадлежит $K^{*}$. Здесь $p^{r}$ обозначает порядок элемента $\sigma$ в соответствующей группе Галуа.

В силу свойств циклических алгебр получаем, что index $K_{\mathfrak{p}}\left[K_{\mathfrak{p}} \otimes_{K} A(K, \chi)\right]=1$ тогда и только тогда, когда $a \in N_{K_{\mathfrak{p}}\left(\varepsilon_{p_{1}}\right) / K_{\mathfrak{p}}}\left(K_{\mathfrak{p}}\left(\varepsilon_{p_{1}^{k_{1}}}\right)\right)$. Заметим, что элемент $a$ равен произведению некоторых корней степени $n$ из единицы. Это означает, что $\nu_{1}(a)=0$, где $\nu_{1}-$ экспоненциальное нормирование поля $K_{\mathfrak{p}}\left(\varepsilon_{p_{1}}^{k_{1}}\right)$. Через $\nu$ обозначим экспоненциальное нормирование поля $K_{\mathfrak{p}}$. Тогда $\nu_{1}=e \cdot \nu$ на поле $K_{\mathfrak{p}}$, где $e$ - индекс ветвления расширения $K_{\mathfrak{p}}\left(\varepsilon_{p_{1}^{k_{1}}}\right) / K_{\mathfrak{p}}$. Поскольку $e \in \mathbb{N}$, то $\nu(a)=0$. Поэтому можно воспользоваться леммой о норменной разрешимости (см. [1, теорема 14.1]) для неразветвленного расширения $K_{\mathfrak{p}}\left(\varepsilon_{p_{1}^{k_{1}}}\right) / K_{\mathfrak{p}}$ и 

элемента $a$, согласно которой $a \in N_{K_{\mathfrak{p}}\left(\varepsilon_{p_{1} k_{1}}\right) / K_{\mathfrak{p}}}\left(K_{\mathfrak{p}}\left(\varepsilon_{p_{1}^{k_{1}}}\right)\right)$. В силу свойств цик-
лических алгебр это означает, что

$$
\operatorname{index}_{K_{\mathfrak{p}}}\left[K_{\mathfrak{p}} \otimes_{K} A(K, \chi)\right]=1 .
$$

Возьмем произвольный простой идеал $P \triangleleft R_{K}$, содержащий $p_{s} \mathbb{Z}$. Чтобы доказать теорему Голдшмидта-Айзекса для случая $p>2$, надо теперь лишь показать, что index $K_{P}\left[K_{P} \otimes_{K} A(K, \chi)\right]=1$.

Заметим, что расширение $K_{P}\left(\varepsilon_{p_{1}^{k_{1}}}\right) / K_{P}$ вполне разветвлено. В частности, в силу равенства $K\left(\varepsilon_{p_{1}^{k_{1}}}\right)=K\left(\varepsilon_{n}\right)$ это означает, что $f\left(K_{P}\left(\varepsilon_{n_{p_{s}^{\prime}}}\right) / K_{P}\right)=1$, но многочлен $x^{n_{p_{s}^{\prime}}}-1 \in \mathbb{F}_{p_{s}}[x]$ является сепарабельным. В силу леммы Гензеля имеем, что $\varepsilon_{n_{p_{s}^{\prime}}} \in K_{P}$. Нам понадобится теорема Фонга, формулировка которой приведена во введении.

Если $p_{s}>2$, то, воспользовавшись теоремой Фонга для нашего случая, получаем $\operatorname{index}_{K_{P}}\left[K_{P} \otimes_{K} A(K, \chi)\right]=1$. Если $p_{s}=2$, то воспользуемся тем (см. $[2$, теорема 4.2.8]), что $m_{\mathbb{Q}_{2}}(\chi) \mid 2$, но в этом случае $\operatorname{Gal}\left(K\left(\varepsilon_{n}\right) / K\right)-p$-группа, причем $p>2$. Будем считать, что $m_{\mathbb{Q}_{2}}(\chi)=2$. Мы знаем, что группа разложения $\operatorname{Gal}\left(K_{P}\left(\varepsilon_{n}\right) / K_{P}\right)$ изоморфно вкладывается в группу $\operatorname{Gal}\left(K\left(\varepsilon_{n}\right) / K\right)$ (см. $\left[5\right.$, предложение 10.3]). Далее, пусть $F_{1}:=\mathbb{Q}_{2}(\chi), F_{2}:=K_{P}(\chi)$. Заметим, что $m_{K_{P}}(\chi)=1$ тогда и только тогда, когда $2 \mid\left(F_{2}: F_{1}\right)$ (см. [1, теорема 32.15$\left.]\right)$. Пусть

$$
2 \nmid\left(F_{2}: F_{1}\right) ;
$$

тогда, так как $m_{K_{P}\left(\varepsilon_{n}\right)}(\chi)=1$, должно быть выполнено условие $2 \mid\left(K_{P}\left(\varepsilon_{n}\right): F_{1}\right)$. Однако число $\left(K_{P}\left(\varepsilon_{n}\right): F_{1}\right)=\left(K_{P}\left(\varepsilon_{n}\right): F_{2}\right)\left(F_{2}: F_{1}\right)$ является нечетным. Это означает, что $m_{K_{P}}(\chi)=1$, или, что то же самое,

$$
\operatorname{index}_{K_{P}}\left[K_{P} \otimes_{K} A(K, \chi)\right]=1 .
$$

Из теоремы Хассе-Брауэра-Нётер-Альберта теперь следует, что $m_{K}(\chi)=1$. Тем самым, теорема 1.3 доказана.

Приведем комментарии к случаю $p=2$.

Из рассмотрения случая $p>2$ следует, что (с учетом тех же обозначений) при $p=2$ достаточно исследовать вопрос об индексе Шура характера $\chi \in \operatorname{Irr} G$ в следующих предположениях, которые в силу доказательства теоремы 1.3 не ограничивают общность:

$$
\varepsilon_{n_{2}^{\prime}} \in K, \quad K \subset \mathbb{Q}\left(\varepsilon_{n}\right), \quad p_{s}=2 .
$$

Действительно, в формулировке теоремы 1.3 для случая $p=2$ присутствует дополнительное условие $\sqrt{-1} \in K$. Если подполе $F$ поля $\mathbb{Q}\left(\varepsilon_{n}\right)$ выбрано так, чтобы $\operatorname{Gal}\left(K\left(\varepsilon_{n}\right) / K\right) \cong \operatorname{Gal}\left(\mathbb{Q}\left(\varepsilon_{n}\right) / F\right)$, то легко видеть, что также $\sqrt{-1} \in F$. Это означает, что $m_{F_{P}}(\chi)=1$ по всем архимедовым пополнениям поля $F$. Мы не будем доказывать теорему 1.3 для случая $p=2$. Вместо этого здесь мы рассмотрим простой пример того, что условие $\sqrt{-1} \in K$ существенно для справедливости теоремы 1.3. Для краткости будем в дальнейшем называть это условие условием сильной невещественности.

ОПРедЕЛЕНИЕ 2.1. Пусть поле $F$, однозначно определяемое по полю $K$, обладает свойством невещественности: $F \nsubseteq \mathbb{R}$. Будем говорить в этом случае, что поле K удовлетворяет условию слабой невещественности. 
Ясно, что условие слабой невещественности нельзя игнорировать, так как существуют конечные группы (доказательство этого факта см. ниже), имеющие неприводимые комплексные представления, не реализуемые в поле вещественных чисел $\mathbb{R}$. Однако выполнения условия слабой невещественности недостаточно для справедливости теоремы 1.3.

ПримеР 2.1. Положим $G:=\mathbb{Z}_{31} \times Q_{8}$, где $Q_{8}$ - группа кватернионов порядка 8. Легко видеть, что $\exp G=124=31 \cdot 4$. Возьмем поле $K:=\mathbb{Q}\left(\varepsilon_{31}\right)$. Тогда для поля $K$ выполнено условие слабой невещественности, но не выполнено условие сильной невещественности. Далее, $\operatorname{Gal}\left(K\left(\varepsilon_{124}\right) / K\right)=\operatorname{Gal}(K(i) / K)$ циклическая группа порядка 2. Покажем, доказав следующую лемму, что найдется характер $\chi \in \operatorname{Irr} G$ такой, что $m_{K}(\chi)=2$.

Лемма 2.1. Существует характер $\psi \in \operatorname{Irr} Q_{8}$ maкой, что $m_{\mathbb{Q}}(\psi)=2$.

ДоказАТЕЛЬСтво. Рассмотрим точное двумерное неприводимое комплексное представление группы $Q_{8}$ в матричной реализации:

$$
Q_{8}=\left\{ \pm\left(\begin{array}{ll}
1 & 0 \\
0 & 1
\end{array}\right), \pm\left(\begin{array}{cc}
i & 0 \\
0 & -i
\end{array}\right), \pm\left(\begin{array}{cc}
0 & 1 \\
-1 & 0
\end{array}\right), \pm\left(\begin{array}{cc}
0 & i \\
i & 0
\end{array}\right)\right\}
$$

Докажем, что указанное представление не реализуется над полем рациональных чисел $\mathbb{Q}$. Пусть это не так. Тогда обозначим через $\left\{U(g) \mid g \in Q_{8}\right\}$ матричное представление над $\mathbb{Q}$. Ясно, что

$$
U(1)=\left(\begin{array}{ll}
1 & 0 \\
0 & 1
\end{array}\right), \quad U(-1)=-U(1), \quad U(i)=\left(\begin{array}{cc}
0 & 1 \\
-1 & 0
\end{array}\right) .
$$

Из условия $i j=-j i$ получим, что

$$
U(j)=\left(\begin{array}{cc}
a & b \\
b & -a
\end{array}\right) .
$$

Поскольку $j^{2}=-1$, то $a^{2}+b^{2}=-1$, но это равенство невозможно над полем $\mathbb{Q}$. Лемма доказана.

Положим $\chi=1_{\mathbb{Z}_{31}} \cdot \psi$, где $\psi \in \operatorname{Irr} Q_{8}$ такой, что $m_{\mathbb{Q}}(\psi)=2$. Докажем, что $m_{K}(\chi)=2$. Легко видеть, что

$$
\min \left\{t \in \mathbb{N}|31|\left(2^{t}-1\right)\right\}=5 .
$$

В силу теоремы Куммера (см. [6, теорема 4.40$])$ можно заключить, что $\left(\mathbb{Q}_{2}\left(\varepsilon_{31}\right)\right.$ : $\left.\mathbb{Q}_{2}\right)=5$. Заметим, что $K_{P} \cong \mathbb{Q}_{2}\left(\varepsilon_{31}\right)$ по определению поля $K$ (здесь $P$ - произвольный простой идеал кольца $R_{K}$, лежащий над $\left.2 \mathbb{Z}\right)$. Теперь достаточно показать, что $m_{K}(\psi)=2$. Заметим, что $\mathbb{Q}(\psi)=\mathbb{Q}$. Из леммы 2.1 и тождества Хассе (см. [1, формула 32.13а]) следует, что $m_{\mathbb{Q}_{2}}(\psi)=2$. Однако если $m_{\mathbb{Q}_{2}\left(\varepsilon_{31}\right)}(\psi)=1$, то $2 \mid 5$, что неверно. Так как $\left(\mathbb{Q}_{2}\left(\varepsilon_{142}\right): \mathbb{Q}_{2}\left(\varepsilon_{31}\right)\right)=2$ в силу теоремы Куммера, то $m_{\mathbb{Q}_{2}\left(\varepsilon_{31}\right)}(\psi)=2$, но тогда $m_{K}(\psi)=2$, что и требовалось показать. Таким образом, пример полностью рассмотрен.

Пример 2.1 показывает, что аналог теоремы Фонга, вообще говоря, неверен (если $\operatorname{Gal}\left(K\left(\varepsilon_{n}\right) / K\right)$ является циклической 2-группой и $K\left(\varepsilon_{n}\right)=K\left(\varepsilon_{n_{2}}\right)$ ). Можно рассмотреть более тонкий вопрос. Так как $\mathbb{Z}_{2^{s}}^{*} \cong\langle-1\rangle_{2} \times\langle 5\rangle_{2^{s-2}}$ при $s \geqslant 2$, условие $\sqrt{-1} \in K$ дает возможность сформулировать следующий аналог теоремы Фонга (так как $i^{5}=i$, то $\operatorname{Gal}\left(K\left(\varepsilon_{n}\right) / K\right) \leqslant\langle 5\rangle_{2^{s-2}}$ с точностью до 
изоморфизма, а $\langle 5\rangle_{2^{s-2}}$ - одна из двух максимальных по порядку циклических подгрупп в $\mathbb{Z}_{2}^{*}$ в случае $\left.s>3\right)$.

ГипотезА. Пусть $G$ - конечная группа порядка $n=2^{s} m, 2 \nmid m$. Возьмем поле нулевой характеристики $K$ такое, чтобы $\varepsilon_{m} \in K$ и $\operatorname{Gal}\left(K\left(\varepsilon_{n}\right) / K\right)$ - циклическая группа порядка больше 2 . Тогда $m_{K}(\chi)=1$ для любого характера $\chi \in \operatorname{Irr} G$.

2.2. Новые достаточные условия. Здесь мы сформулируем и докажем новые достаточные условия, которые делают справедливой теорему Голдшмидта-Айзекса для случая $p=2$. Как было отмечено, условие сильной невещественности обеспечивает справедливость утверждения. Также на примере 2.1 было установлено, что условия слабой невещественности недостаточно. Тем не менее, ясно, что условие слабой невещественности в некотором смысле необходимо для справедливости утверждения "равномерно" по всем конечным группам экспоненты $n$. Поэтому новые достаточные условия направлены на смягчение сильного условия невещественности, которое налагает достаточно строгие ограничения на поле $K$.

2.2.1. Алгоритмический признак. Как было видно из доказательства теоремы 1.3, наиболее сложный случай возникал при $p=2$ и $p_{s}=2$. Формулируемый ниже алгоритмический признак гарантирует выполнение условия $p_{s}>2$.

Заметим, что в силу китайской теоремы об остатках справедливо разложение

$$
\mathbb{Z}_{n}^{*}=A_{1} \times \cdots \times A_{s}
$$

где $n=p_{1}^{k_{1}} \cdots p_{s}^{k_{s}}, A_{i} \cong \mathbb{Z}_{p_{i}^{*}}^{*}$.

TЕОРема 2.1 (алгоритмический признак). Пусть $G$ - конечная группа экспоненты $n$. Пусть также $K$ - произвольное поле нулевой характеристики, удовлетворяющее условию слабой невещественности, причем $\operatorname{Gal}\left(K\left(\varepsilon_{n}\right) / K\right)$ является ииклической 2-группой с условием $\operatorname{Gal}\left(K\left(\varepsilon_{n}\right) / K\right) \nsubseteq \mathbb{Z}_{2^{\nu_{2}(n)}}^{*}$, где $\mathbb{Z}_{2^{\nu_{2}(n)}}^{*} \cong A_{2^{\nu_{2}(n)}}$. Тогда для любого $\chi \in \operatorname{Irr} G$ выполнено $m_{K}(\chi)=1$.

ДокАзАтЕльство. Если доказать, что $K\left(\varepsilon_{n}\right) \neq K\left(\varepsilon_{2^{\nu_{2}(n)}}\right)$, то в силу доказательства теоремы 1.3 получим искомое утверждение. Для удобства обозначим $k:=\nu_{2}(n)$. Условие $K\left(\varepsilon_{n}\right) \neq K\left(\varepsilon_{2^{k}}\right)$ эквивалентно тому, что найдется некоторый неединичный автоморфизм $\sigma \in \operatorname{Gal}\left(K\left(\varepsilon_{n}\right) / K\right)$ такой, что $\varepsilon_{2^{k}}^{\sigma}=\varepsilon_{2^{k}}$. Поскольку $\varepsilon_{2^{k}}=\varepsilon_{n}^{n_{2^{\prime}}}$, для выполнения условия $K\left(\varepsilon_{n}\right) \neq K\left(\varepsilon_{2^{k}}\right)$ необходимо и достаточно, чтобы нашлось некоторое число $t \in \mathbb{Z}_{\geqslant 2}$ (лежащее с точностью до изоморфизма в группе $\left.\operatorname{Gal}\left(K\left(\varepsilon_{n}\right) / K\right)\right)$, взаимно простое с $n$, причем $\varepsilon_{n}^{t n_{2^{\prime}}}=\varepsilon_{n^{\prime}}^{n_{2^{\prime}}}$. Ясно, что это выполнено тогда и только тогда, когда

$$
n\left|\left((t-1) n_{2^{\prime}}\right) \Longleftrightarrow 2^{k}\right|(t-1) .
$$

Если $\operatorname{Gal}\left(K\left(\varepsilon_{n}\right) / K\right) \nsubseteq \mathbb{Z}_{2^{k}}^{*}$, то можно отождествить $\mathbb{Z}_{2^{k}}^{*}$ с подгруппой в $\mathbb{Z}_{n}^{*}$, но это означает, что найдется число $t \in \operatorname{Gal}\left(K\left(\varepsilon_{n}\right) / K\right)$ (после проведения всех отождествлений) такое, что $t \equiv 1\left(\bmod 2^{k}\right)$, откуда следует требуемое утверждение.

Особенностью алгоритмического признака является его эффективность, т.е. возможность проверить с помощью простого алгоритма. Более того, при 
выполнении без ограничения общности условия слабой невещественности можно считать, что $\operatorname{Gal}\left(K\left(\varepsilon_{n}\right) / K\right) \cong \mathbb{Z}_{2^{\nu_{2}(n)}}^{*}$ (это следует из доказательства теоремы Голдшмидта-Айзекса, в силу которого при выполнении условия слабой невещественности можно считать, что $\left.\varepsilon_{n_{p_{s}^{\prime}}} \in K, K\left(\varepsilon_{n}\right)=K\left(\varepsilon_{p_{1}^{k_{1}}}\right)\right)$.

2.2.2. Другой достаточный признак. Сформулируем более абстрактную теорему о достаточных условиях применимости теоремы 1.3.

Теорема 2.2. Пусть $G$ - конечная группа экспоненты $n$. Пусть, далее, поле нулевой характеристики $K$ удовлетворяет условию слабой невещественности, причем $\operatorname{Gal}\left(K\left(\varepsilon_{n}\right) / K\right)$ - циклическая 2-группа. Если

$$
\nu_{2}\left(\min \left\{t \in \mathbb{N}: n_{2^{\prime}} \mid\left(2^{t}-1\right)\right\}\right)=\nu_{2}\left(\varphi\left(n_{2^{\prime}}\right)\right),
$$

то для любого $\chi \in \operatorname{Irr} G$ выполнено $m_{K}(\chi)=1$.

ДокАЗАТЕльство. Если считать, что выполнен алгоритмический признак, то утверждение теоремы тривиально. Поэтому будем считать, что алгоритмический признак не выполнен, но тогда можно считать без ограничения общности, что

$$
\varepsilon_{n_{2^{\prime}}} \in K, \quad K\left(\varepsilon_{n}\right)=K\left(\varepsilon_{n_{2}}\right) .
$$

По теореме Куммера (см. [6, теорема 4.40]) расширение $\mathbb{Q}_{2}\left(\varepsilon_{n_{2^{\prime}}}\right) / \mathbb{Q}_{2}$ неразветвлено. Условие теоремы означает, что число $\frac{\left(\mathbb{Q}\left(\varepsilon_{n_{2^{\prime}}}\right): \mathbb{Q}\right)}{\left(\mathbb{Q}_{2}\left(\varepsilon_{n_{2^{\prime}}}\right): \mathbb{Q}_{2}\right)}$ нечетно. Выберем поле $F$ так, чтобы

$$
\mathbb{Q} \subset F \subset \mathbb{Q}\left(\varepsilon_{n}\right),
$$

причем

$$
\operatorname{Gal}\left(K\left(\varepsilon_{n}\right) / K\right) \cong \operatorname{Gal}\left(\mathbb{Q}\left(\varepsilon_{n}\right) / F\right) .
$$

Тогда число простых идеалов кольца $R_{F}(\chi)$ целых алгебраических чисел поля $F(\chi)$, лежащих над $2 \mathbb{Z}$, нечетно (так как $\mathbb{Q}\left(\varepsilon_{n_{2^{\prime}}}\right) \subset F(\chi) \subset \mathbb{Q}\left(\varepsilon_{n}\right)$, по теореме Куммера любое 2 -адическое пополнение расширения $F(\chi) / \mathbb{Q}\left(\varepsilon_{n_{2}^{\prime}}\right)$ является вполне разветвленным, поэтому число простых идеалов кольца $R_{F(\chi)}$, лежащих над $2 \mathbb{Z}$, равно числу простых идеалов кольца $R_{\mathbb{Q}\left(\varepsilon_{n_{2^{\prime}}}\right)}$, лежащих над $\left.2 \mathbb{Z}\right)$. Результат теоремы теперь следует из доказательства теоремы ГолдшмидтаАйзекса, невещественности поля $F$ и тождества Хассе (если $m_{K}(\chi) \neq 1$, то $m_{K}(\chi)=2$ в силу доказательства теоремы Голдшмидта-Айзекса, но число нетривиальных инвариантов Хассе нечетно и все они равны $\frac{1}{2}$ в силу теоремы Бенарда-Шахера; тем самым, $\left.m_{K}(\chi)=1\right)$.

Заметим, что если $G$ - конечная 2-группа, то условия слабой невещественности, наложенного на поле $K$, достаточно для справедливости теоремы 2.2. Это следует из доказательства теоремы Шиллинга-Витта-Рокетта, проведенного в [1, теорема 41.9].

2.2.3. Оценка индекса Шура над полем $\mathbb{Q}$. Наша ближайшая цель состоит в том, чтобы дать практический алгоритм построения поля алгебраических чисел $K \subset \mathbb{Q}\left(\varepsilon_{n}\right)$, для которого $\operatorname{Gal}\left(K\left(\varepsilon_{n}\right) / K\right)$ - циклическая подгруппа в $\operatorname{Gal}\left(\mathbb{Q}\left(\varepsilon_{n}\right) / \mathbb{Q}\right) \cong \mathbb{Z}_{n}^{*}$ максимального порядка, причем для поля $F$, отвечающего силовской 2-подгруппе в $\operatorname{Gal}\left(K\left(\varepsilon_{n}\right) / K\right)$, выполнены некоторые достаточные 
условия применимости теоремы 1.3. Данное построение дает оценку индекса Шура произвольного комплексного неприводимого характера произвольной конечной группы экспоненты $n$.

Теорема 2.3 (об оценке). Пусть $G$ - конечная группа порядка (или экспоненты) $n$. Пусть $m$ - порядок какой-нибудь максимальной ииклической подгруппы в группе $\mathbb{Z}_{n}^{*}$. Если $n \neq 4$, mо $m_{\mathbb{Q}}(\chi) \mid \frac{\varphi(n)}{m}$ для любого $\chi \in \operatorname{Irr} G$.

ДокАЗАтЕльство. Разобьем доказательство на несколько шагов.

Шаг 1. Заметим, что если $G-p$-группа нечетного порядка, то результат тривиально следует из теоремы Шиллинга-Витта-Рокетта. Поэтому считаем, что группа $G$ не лежит в классе $p$-групп нечетного порядка. Сначала выберем специальным образом некоторую максимальную циклическую подгруппу в группе $\mathbb{Z}_{n}^{*}$. Пусть $n=p_{1}^{k_{1}} \cdots p_{s}^{k_{s}}$ - разложение числа $n$ на простые множители. Тогда имеет место прямое разложение $\mathbb{Z}_{n}^{*}=A_{1} \times \cdots \times A_{s}$, где $A_{i} \cong \mathbb{Z}_{p_{i}^{*}}^{*}$. Если $2 \nmid n$, то можно выбрать максимальную циклическую подгруппу в $\mathbb{Z}_{n}^{*}$ произвольно. Обозначим ее $M$.

Пусть $2 \mid n$. Для определенности будем считать, что $A_{1} \cong \mathbb{Z}_{2^{\nu_{2}(n)}}^{*}$. Тогда максимальную циклическую подгруппу будем выбирать следующим образом. Для некоторого простого $p>2$ возьмем в каждой группе $A_{i}$ при $i>1$ по силовской p-подгруппе. Среди всех таких подгрупп возьмем максимальную по порядку (если таких несколько, то берем произвольную). Далее проделаем ту же процедуру для другого простого числа $q>2$. Пусть $M_{p}, M_{q}$ - выбранные таким образом подгруппы. Тогда положим $M_{p q}:=M_{p} \times M_{q}$. Ясно, что группа $M_{p q}$ является циклической. Выбираем затем еще одну максимальную силовскую $h$-подгруппу для нечетного простого $h \notin\{p, q\}$ среди силовских $h$-подгрупп в группах $A_{i}$ для $i=\overline{2, s}$ (если их несколько, то берем произвольную) и добавляем с помощью конструкции прямого произведения к группе $M_{p q}$. Повторим эту процедуру для всех нечетных простых. В итоге мы получим некоторую максимальную циклическую 2 -подгруппу в группе $\mathbb{Z}_{n}^{*}$.

Теперь выберем в каждой из групп $A_{i}, i=\overline{2, s}$, по силовской 2-подгруппе. Из выбранных силовских 2-подгрупп возьмем максимальную по порядку. Обозначим искомую подгруппу $\bar{M}_{2}$.

Наконец, рассмотрим группу $A_{1} \cong \mathbb{Z}_{2^{\nu_{2}(n)}}^{*}$. Обозначим $t:=\nu_{2}(n)$. Пусть сначала $t \geqslant 3$, тогда $A_{1} \cong\langle-1\rangle_{2} \times\langle 5\rangle_{2^{t-2}}$. Обозначим через $\bar{M}$ образ подгруппы $\langle 5\rangle_{2^{t-2}}$ группы $\mathbb{Z}_{2^{t}}^{*}$ в группе $A_{1}$ при изоморфизме. Если $t=\overline{1,2}$, то полагаем $\bar{M}=\{1\}$. Сравним порядки групп $\bar{M}_{2}$ и $M$. Положим $M_{2}:=$ $\operatorname{argmax}\left\{\left|\bar{M}_{2}\right|,|\bar{M}|\right\}$. Положим $M:=M_{2^{\prime}} \times M_{2}$, где $M_{2^{\prime}}:=\prod_{i=2}^{s} M_{p_{i}}$. Напомним, что мы положили $p_{1}:=2$, если $2 \mid n$.

Итак, мы выбрали специальным образом максимальную циклическую подгруппу $M$ в $\mathbb{Z}_{n}^{*}$. Единственный случай, когда мы не смогли сделать выбор именно максимальной циклической подгруппы в $\mathbb{Z}_{n}^{*}$, состоит в предположении, что либо $n=1$, либо $n=2$, либо $n=4$. Если $n \mid 2$, то утверждение теоремы тривиально. По условию теоремы случай $n=4$ не может возникнуть.

Шаг 2. Выберем $K$ так, чтобы $\mathbb{Q} \subset K \subset \mathbb{Q}\left(\varepsilon_{n}\right)$, причем $\operatorname{Gal}\left(\mathbb{Q}\left(\varepsilon_{n}\right) / K\right) \cong M$. Докажем, что для любого характера $\chi \in \operatorname{Irr} G$ выполнено $m_{K}(\chi)=1$.

Если $m_{K}(\chi)$ является нечетным числом, то, выбрав произвольный простой делитель $p$ числа $m_{K}(\chi)$, силовскую $p$-подгруппу в $\operatorname{Gal}\left(\mathbb{Q}\left(\varepsilon_{n}\right) / K\right)$ и поле $F$, 
ей соответствующее по фундаментальному соответствию Галуа, получим противоречие с теоремой Голдшмидта-Айзекса в случае $p>2$. Аналогично рассуждая в случае четности числа $m_{K}(\chi)$, получаем, что число $m_{K}(\chi)$ является степенью двойки (можно даже показать, что $m_{K}(\chi)=2$, но это нам не понадобится). Приведем это утверждение к противоречию. Для этого возьмем силовскую 2 -подгруппу в группе $\operatorname{Gal}\left(\mathbb{Q}\left(\varepsilon_{n}\right) / K\right)$ и поле $F$, соответствующее ей по фундаментальному соответствию Галуа. По построению $M_{2}=\operatorname{Gal}\left(\mathbb{Q}\left(\varepsilon_{n}\right) / F\right)$. Достаточно теперь доказать, что $m_{F}(\chi)=1$. Чтобы применить теорему Голдшмидта-Айзекса, необходимо проверить достаточные условия.

Пусть группа $M_{2}$ была выбрана в качестве подгруппы в группе $A_{i}$ для некоторого $i=\overline{2, s}$. Заметим тогда, что $M_{2} \nsubseteq A_{1}$, так как группы $A_{i}, A_{1}$ тривиально пересекаются, а порядок группы $M_{2}$ больше единицы. Без ограничения общности слабое условие невещественности для поля $F$ можно считать выполненным. Действительно, если $s>2$, то поле $F$ по своему построению содержит прими-

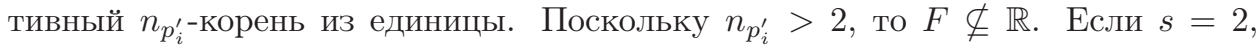
то слабое условие невещественности необходимо проверить лишь для случая $n=2 p^{\alpha}$, где $p$ - некоторое нечетное простое число. Разберем этот случай подробно в шаге 4. Здесь же будем считать, что он не имеет места. Применим алгоритмический признак, по которому $m_{F}(\chi)=1$.

Пусть группа $M_{2}$ была выбрана в качестве подгруппы в группе $A_{1}$. Если $n>4$, то группа $M_{2}$ с точностью до изоморфизма является подгруппой в $\langle 5\rangle_{2^{t-2}}$. Заметим, что $i^{5}=i$, но это означает, что $i \in F$. Тем самым, выполнено сильное условие невещественности, поэтому $m_{F}(\chi)=1$ по теореме Голдшмидта-Айзекса. Случаи $n \mid 4$ были рассмотрены в шаге 1. Заметим только, что в этих исключительных случаях $F=\mathbb{Q}\left(\varepsilon_{n}\right)$ в силу выбора группы $M_{2}$. Поэтому также будет $m_{F}(\chi)=1$.

Шаг 3. Положим $k:=|M|, m:=\frac{\varphi(n)}{k}$. Нам осталось доказать оценку $m_{\mathbb{Q}}(\chi) \mid m$. Из шага 2 следует, что операторное представление группы $G$ с характером $\chi$ реализуется с помощью некоторого матричного представления в поле $K(\chi)$. Более того, из классической теории индекса Шура следует, что $m_{\mathbb{Q}(\chi)}(\chi) \mid(K(\chi): \mathbb{Q}(\chi))$. Поскольку по определению $m_{\mathbb{Q}}(\chi)=m_{\mathbb{Q}(\chi)}(\chi)$, для справедливости оценки достаточно установить, что $(K(\chi): \mathbb{Q}(\chi)) \mid m$. Для этого обозначим $T:=K \cap \mathbb{Q}(\chi), E:=K(\chi)=K \cdot \mathbb{Q}(\chi)$. Из доказательства теоремы об ограничении для скрещенных алгебр (см. [1, теорема 29.13]) имеем изоморфизм групп Галуа $\operatorname{Gal}(E / \mathbb{Q}(\chi)) \cong \operatorname{Gal}(K / T)$, но число $(K(\chi): \mathbb{Q}(\chi))$ равно порядку группы $\operatorname{Gal}(E / \mathbb{Q}(\chi))$. Заметим теперь, что число $m$ равно порядку группы $\operatorname{Gal}(K / \mathbb{Q})$, которая содержит в качестве подгруппы группу $\operatorname{Gal}(K / T)$. Результат теперь следует из теоремы о размерности короткой башни полей.

Шаг 4. Осталось разобрать случай, когда $n=2 p^{\alpha}$ для некоторого нечетного простого числа $p$. Возьмем любой характер $\chi \in \operatorname{Irr} G$ и докажем для него, что $m_{\mathbb{Q}}(\chi)=1$. Заметим, что группа $\mathbb{Z}_{n}^{*}$ является циклической. Более того, $\mathbb{Q}\left(\varepsilon_{n}\right)=\mathbb{Q}\left(\varepsilon_{p^{\alpha}}\right)$. Это означает, что применима теорема Фонга, по которой индекс Шура $m_{\mathbb{Q}}(\chi)$ равен единице. Теорема доказана.

Заметим, что полученная оценка "равномерна" по всем конечным группам экспоненты $n$ (или по всем группам порядка $n$ ) и не зависит, в частности, от поля комплексных характеров и размерности комплексных неприводи- 
мых представлений. Более того, эта оценка чрезвычайно просто вычисляется на основании лишь числа $\varphi(n)$. Также видно, что на практике нет необходимости проверять какие-либо достаточные условия применимости теоремы Голдшмидта-Айзекса.

\section{§ 3. Заключение}

В 2011 г. исполняется 200 лет со дня рождения Э. Галуа. В связи с этим хочется закончить работу следующей теоремой.

Теорема 3.1. Рассмотрим конечную группу $G$ нечетного порядка $n$. Пусть, далее, $n=p_{1}^{k_{1}} \cdots p_{s}^{k_{s}}-$ каноническое разложение числа $n$ на простые множители. Обозначим $m=p_{1} \cdots p_{s}$. Если правилъный $m$-угольник допускает построение с помощью ииркуля и линейки, то для любого характера $\chi \in \operatorname{Irr} G$ имеем $m_{\mathbb{Q}}(\chi)=1$.

ДокАЗАТЕЛЬство. Из теории Галуа следует, что правильный $m$-угольник допускает построение циркулем и линейкой тогда и только тогда, когда размерность $\left(\mathbb{Q}\left(\varepsilon_{m}\right): \mathbb{Q}\right)$ является степенью двойки. По условию теоремы число $m$ нечетно, откуда простые числа $p_{1}, \ldots, p_{s}$ являются простыми числами Ферма, т. е. элементами множества

$$
\left\{2^{2^{k}}+1 \mid k \in \mathbb{Z}_{\geqslant 0}, 2^{2^{k}}+1 \text { простое }\right\} .
$$

Фиксируем произвольный характер $\chi \in \operatorname{Irr} G$. Предположим, что $m_{\mathbb{Q}}(\chi)>1$. Заметим, что $m_{\mathbb{Q}}(\chi)=\operatorname{HOK}\left\{m_{\mathbb{Q}_{p_{i}}}(\chi) \mid i=\overline{1, s}\right\}$. Далее, из [2, теорема 4.2.8] следует, что

$$
m_{\mathbb{Q}_{p_{i}}}(\chi) \mid\left(p_{i}-1\right) \quad \forall i=\overline{1, s} .
$$

Тогда заведомо $2 \mid m_{\mathbb{Q}}(\chi)$. Получаем противоречие с нечетностью числа $n$.

\section{Список литературы}

1. I. Reiner, Maximal orders, London Math. Soc. Monogr. (N.S.), 28, Oxford Univ. Press, Oxford, 2003.

2. С. Д. Берман, "Представления конечных групп над произвольным полем и над кольцами целых чисел", Изв. АН СССР. Сер. матем., 30:1 (1966), 69-132.

3. D. M. Goldschmidt, I. M. Isaacs, "Schur indices in finite groups", J. Algebra, 33 (1975), 191-199.

4. Ch. W. Curtis, I. Reiner, Methods of representation theory, v. 2, Pure Appl. Math. (N. Y.), Wiley, New York, 1987.

5. Д. Касселс, А. Фрёлих, Алгебрачческая теория чисел, Мир, М., 1969; пер. с англ.: J. W. S. Cassels, A. Fröhlich, Algebraic number theory, Academic Press, London, 1967.

6. Ch. W. Curtis, I. Reiner, Methods of representation theory, v. 1, Wiley, New York, 1981.

Д. Д. Киселев (D. D. Kiselev)

Московский государственный университет им. М. В. Ломоносова, механико-математический факультет

E-mail: denmexmath@yandex.ru
Поступило в редакцию 01.03 .2011 18.05 .2012 